\title{
Laboreal
}

Volume $17 \mathrm{~N}^{\circ} 1$ | 2021

Trabalhar hoje: mudanças, permanências, estratégias, reinvenções

\section{1 de março de 2020: o trabalho na Atenção Primária à Saúde (APS) interpelado pela pandemia da Covid-19}

11 de marzo de 2020: el trabajo en la Atención Primaria de Salud (APS)

interpelado por la pandemia de Covid-19

11 mars 2020: les travaux dans les soins de santé primaires (SSP) interpeller par la pandémie Covid-19

March 11, 2020: work in Primary Health Care (PHC) challenged by the Covid-19 pandemic

\section{Ana Cláudia Barbosa da Silva-Roosli}

\section{OpenEdition}

\section{Journals}

\section{Edição electrónica}

URL: https://journals.openedition.org/laboreal/17693

ISSN: 1646-5237

\section{Editora}

Universidade do Porto

\section{Refêrencia eletrónica}

Ana Cláudia Barbosa da Silva-Roosli, «11 de março de 2020: o trabalho na Atenção Primária à Saúde (APS) interpelado pela pandemia da Covid-19», Laboreal [Online], Volume $17 \mathrm{~N}^{0} 1$ | 2021, posto online no dia 18 junho 2021, consultado o 20 junho 2021. URL: http://journals.openedition.org/laboreal/ 17693

Este documento foi criado de forma automática no dia 20 junho 2021.

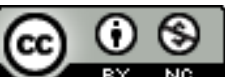

Laboreal está licenciado com uma Licença Creative Commons - Atribuição-NãoComercial 4.0 Internacional. 


\title{
11 de março de 2020: o trabalho na Atenção Primária à Saúde (APS) interpelado pela pandemia da Covid-19
}

\author{
11 de marzo de 2020: el trabajo en la Atención Primaria de Salud (APS) \\ interpelado por la pandemia de Covid-19 \\ 11 mars 2020: les travaux dans les soins de santé primaires (SSP) interpeller par \\ la pandémie Covid-19 \\ March 11, 2020: work in Primary Health Care (PHC) challenged by the Covid-19 \\ pandemic
}

Ana Cláudia Barbosa da Silva-Roosli

\section{Introdução}

1 Desde 11 de março de 2020, com o reconhecimento da pandemia de Covid-19 pela Organização Mundial de Saúde (OMS), há um esforço global para contê-la, e assim impedir o crescimento de doentes e mortes. No plano do trabalho em saúde, a evolução da Covid-19 demanda diversificados pontos de intervenção, a fim de assistir doentes com diferentes necessidades, fases da infecção e espectro de gravidade: desde "o monitoramento de casos leves em isolamento domiciliar, com orientações para o manejo de sintomas e para a identificação precoce de sinais de alerta, até a internação em unidades de terapia intensiva (UTI) e a reabilitação após a alta hospitalar" (Daumas et al., 2020, p. 1).

De forma desigual, esta diversidade pode ser abarcada pelo sistema de saúde brasileiro, composto por uma combinação entre o sistema público - o Sistema Único de Saúde (SUS) - e um subsistema privado, majoritariamente orientado para a oferta de assistência médico-hospitalar (Teixeira et al., 2020). O SUS, criado em 1988 com o 
estabelecimento constitucional da saúde como um direito, norteia-se pelos princípios da universalidade e da integralidade.

3 O Brasil é o único país do mundo com um sistema universal público de saúde para uma população de mais de 210 milhões de habitantes (Guimarães, 2020). Atualmente dependem exclusivamente do SUS 162 milhões de pessoas, para qualquer atendimento. $\mathrm{Na}$ outra ponta, 47 milhões de pessoas, com maior renda, adquirem voluntariamente planos de saúde privados. Mesmo assim, em algum momento, esta fatia da população também faz uso do SUS pelas ações da vigilância sanitária, de vacinação em massa ou dos programas públicos de transplante, por exemplo (Dantas, 2020).

4 A elevada taxa de letalidade da Covid-19 levou o Brasil, como a maioria dos países do mundo, a centralizar sua resposta à pandemia no trabalho em saúde operado na assistência hospitalar, no manejo médico individual e desenvolvimento de ações curativas (Medina, Giovanella, Bousquat, Mendonça, \& Aquino, 2020). Embora importante e necessária, é possível elencar várias limitações desta diretriz. A começar pela cobertura de leitos hospitalares do país, atendendo apenas $1 / 4$ da população brasileira (Teixeira et al., 2020), com concentração de mais de $2 / 3$ destes no sistema privado de saúde.

5 Nesta direção, a ênfase recaiu no manejo clínico do novo coronavírus e no aprendizado de protocolos para medidas individuais (higiene e uso de equipamentos de proteção) em detrimento de uma discussão em torno das condições necessárias à realização do trabalho. Há relatos de profissionais sobre condições precarizadas, higiene inadequada, jornadas extenuantes, falta de treinamento e insuficiência ou indisponibilidade de equipamentos de proteção, mesmo nos serviços de terapia intensiva (Jackson et al., 2020). Os índices entre os trabalhadores da saúde parecem atestar esta realidade: são mais de 484 mil casos de adoecimento por Covid-19 (COFEN - Conselho Federal de Enfermagem, 2021b) e pelo menos 990 óbitos (Schmitt, 2021). Entre profissionais de enfermagem, o país responde por um terço das mortes globais pelo vírus (COFEN, 2021a).

6 A estratégia priorizada no Brasil negligencia outra dimensão da Covid-19: trata-se de "uma doença comunitária, que se dispersa muito rapidamente e se manifesta de forma similar a outras síndromes gripais (SG), sendo de difícil contenção" (Teixeira et al., 2020, p. 1), o que torna cruciais ações de vigilância à saúde com testagem, detecção do maior número de casos, rastreamento e monitoramento de contactantes. A epidemiologista Fassa (2021, p. 19) é categórica: "uma área em que estamos errando muito é na vigilância" e defende, juntamente a diversos pesquisadores (Medina et al., 2021), seu desenvolvimento e/ou articulação com a Atenção Primária à Saúde (APS) para minimizar danos e reduzir as mortes evitáveis.

7 Enfrentar a pandemia apoiando-se no trabalho em saúde no SUS exige uma dupla tarefa: a superação dos limites por sua condição real - dificuldades para gerir suas demandas cotidianas, insuficiente infraestrutura da rede hospitalar - e sua reorganização na resposta ao quadro sanitário instalado. 


\section{As potencialidades do trabalho na APS na luta contra a pandemia no Brasil}

8 A APS possui ampla estrutura, com unidades de saúde distribuídas pelo país, atingindo até as regiões mais desprovidas de outros recursos; o que lhe confere uma cobertura estimada de $65 \%$ da população nacional (Giovanella et al., 2021). Suas atribuições prescritas orientam que o trabalho se dê de forma territorializada, com ações no âmbito individual e coletivo, contemplando a promoção, a prevenção, o diagnóstico, o tratamento, a reabilitação e a redução de danos.

9 Nesta crise, o trabalho na APS pode tanto dirigir-se a cidadãos não doentes, quanto orientar-se para a proteção do contágio, detecção precoce dos sintomáticos, rastreamento dos contatos, isolamento, tratamento (Medina et al., 2020) e reabilitação de pacientes com sequelas da Covid-19. Também pode intervir junto a grupos sociais vulneráveis, identificar situações de extrema precariedade e articular ações intersetoriais que colaborem para a "segurança epidêmica, alimentar e de outras ordens, para contingentes populacionais cada vez mais empobrecidos" (Seixas et al., 2021, p. 8).

10 Enquanto estratégia para reduzir o espraiamento da pandemia, o trabalho na APS também pode se beneficiar de sua histórica e bem-sucedida experiência na execução do Programa Nacional de Imunizações. Dimensão particularmente importante, tanto por causa da disponibilidade de diferentes vacinas contra a Covid-19 e seus diferentes intervalos entre as doses, quanto por um fato que pode comprometer a proteção da vacinação no Brasil: até o momento, mais de meio milhão de pessoas que receberam a primeira dose da Coronavac, principal vacina aplicada no país, não retornaram para receber a segunda dose, cujo prazo deveria ser até 28 dias após a primeira (Gamba \& Righetti, 2021).

11 A política central de expansão da APS é a Estratégia de Saúde da Família (ESF), cujo processo de trabalho envolve uma equipe multiprofissional, composta por médicos, enfermeiros, auxiliares de enfermagem e o agente comunitário de saúde (ACS), categoria profissional existente exclusivamente no SUS. Em março de 2020 eram contabilizadas " 44 mil equipes da ESF e 260 mil ACS distribuídas pelos 5.560 municípios do país" (Giovanella et al., 2021, pp. 14-15).

12 Nesse momento, pensamos que o trabalho dos ACS deveria estar em relevo: seu pilar é a estreita relação com os usuários e sua essencialidade está na educação em saúde, acompanhada de registro e produção de informações para diagnóstico territorial, mapeamento de demandas e peculiaridades do território sob sua responsabilidade, principalmente por meio da visita domiciliar (VD) (Portaria nํำ.436, de 21 de setembro de 2017). Contudo, diversos estudos indicam uma histórica falta de reconhecimento do trabalho do ACS; corroborada pela subestimação do seu papel no enfrentamento da pandemia, gerando, por exemplo, uma distribuição desigual de equipamentos de proteção individual (EPI) em prejuízo desta categoria (Nogueira et al., 2020). É importante ressaltar ainda que, embora seja evidente que as atribuições da APS são fundamentais para o enfrentamento da pandemia, sua potência não se limita às prescrições já sinalizadas. Ao almejar o autocuidado em saúde, no cerne do trabalho na APS está a relação de serviço, que se caracteriza por produzir uma transformação nas condições de atividade e nas disposições de ação do usuário/cliente (Zarifian, 2001). 
Frente ao grave quadro epidemiológico, o autocuidado também deveria se assentar na solidariedade, na expectativa de que seus efeitos transcendam a esfera individual e privada dos usuários, produzindo repercussões positivas na vida comunitária.

Por depender da apropriação pelo usuário/cliente daquilo que lhe é oferecido, a produção do serviço sustenta-se na mobilização das competências profissionais para identificar e conhecer a atividade do usuário/cliente, interpretar e compreender o seu ponto de vista, mobilizar recursos e agir com pertinência sobre as suas condições de execução (Zarifian, 2001). Desse modo, a produção do serviço extrapola o momento da interação e demanda a mobilização da atividade, individual e coletiva, num contexto sociotécnico (Salini, Jaramillo, Goudeaux, \& Poizat, 2018).

Neste sentido, sublinha-se traço característico do trabalho em saúde na APS: é aberto aos modos de vida parcialmente imprevistos dos usuários/pacientes, o que impede a antecipação total das ações de saúde e exige um recuo das normas prescritas. Na APS, o trabalho em saúde demanda uma postura muito mais compreensiva do que prescritiva, e os modos de atingir os objetivos e resultados são particularmente dependentes da iniciativa dos trabalhadores.

Há, então, um convite incontornável: interrogar o trabalho em saúde na APS a partir da atividade mobilizada por seus trabalhadores. Em meio a tantas lacunas sobre a Covid-19, deixar-se interpelar pela atividade de trabalho significa dar visibilidade aos debates entre as normas antecedentes e as tendências de renormatização dos trabalhadores (Schwartz, Adriano, \& Abderrahmane, 2008), buscando capitalizar saberes e escolhas confeccionadas nas tentativas de mitigação dos efeitos de saúde e sociais da pandemia no território brasileiro, acessando eficácias potencialmente agenciáveis em patrimônios coletivos.

Este encaminhamento também permite dar visibilidade ao diálogo com os valores circulantes nos territórios, analisar os possíveis e impossíveis para o deslizamento e afirmação na direção dos valores orientados ao bem comum. Questão de absoluta importância numa sociedade tão marcada pela banalização da violência e da morte, como a brasileira. Trata-se, portanto, de ir além da denúncia das ações governamentais no gerenciamento da crise sanitária, que transitam, no mínimo, entre a omissão e a desorganização.

17 A interpelação do trabalho em saúde pelo ponto de vista da atividade deve orientar a compreensão tanto dos recursos quanto dos obstáculos à ação dos trabalhadores, a fim de respaldar a oferta de condições objetivas favoráveis ao desenvolvimento e à consolidação de competências e saberes tecidos na aderência dos territórios (Schwartz et al., 2008).

18 Enfim, potencialidades do trabalho na APS têm sido desperdiçadas. Apesar do cenário prévio à pandemia - marcado pela falta de investimentos, pelo histórico subfinanciamento do SUS e pelo novo modelo de financiamento da APS (Daumas et al., 2020) - aliado à inexistência de uma coordenação nacional da pandemia no SUS, foram ensaiadas algumas iniciativas que investiram na reorganização do trabalho em saúde na APS, como se verá a seguir. 


\section{Reorganizações do trabalho na APS durante a pandemia}

19 No Brasil, um encaminhamento comum da organização da atenção à saúde foi o fechamento de unidades e redirecionamento de equipes para polos de atendimento e atenção para pacientes com sintomas respiratórios, gerando a desarticulação da assistência à saúde aos usuários sob sua responsabilidade nos territórios.

Em outra via, alternativas de resposta à pandemia na APS desenvolveram-se de forma integrada à Vigilância Epidemiológica, algumas destacadas por sua potencialidade na produção de impacto positivo na luta contra o novo coronavírus. Desse modo, e mediante exigências de distanciamento físico, foram desenvolvidas algumas experiências de reorganização do trabalho pautadas pela telessaúde, com prestação de serviços de saúde à distância e intermediados por tecnologias da informação e comunicação (TICs).

21 Por exemplo, na busca por manter a oferta de ações de saúde à população como um todo, Unidades de APS das cidades de Atalaia (AL), Rio de Janeiro (RJ) e Vilhena (RO) reorientaram seus fluxos e o processo de trabalho integrando a continuidade do cuidado a portadores de agravos crônicos ao atendimento de casos suspeitos de Covid-19. Associaram o telemonitoramento e a vigilância de casos de Covid-19 no território ao telemonitoramento/teleconsultas e visitas domiciliares dirigidas aos usuários em situação de vulnerabilidade (Favoreto, 2020).

Nos municípios de Atalaia (AL) e do Rio de Janeiro (RJ), Favoreto (2020) destacou ainda a educação permanente de todos os integrantes da equipe de SF e a mobilização comunitária, com o envolvimento de lideranças e organizações locais nas intervenções de educação e promoção à saúde, através do uso de mídias sociais, como grupos de WhatsApp e vídeo-chamadas. Em Vilhena (RO), na região norte do Brasil, Riva, Moreira, Silva e Oliveira, (2020, p. 24) avaliaram que as ações realizadas "reduziram drasticamente as formas de contágio da Covid-19 nas Unidades Básicas de Saúde (UBS) ou no contexto domiciliar", consolidando a ESF.

No município de Londrina (PR), uma equipe de Residência Multiprofissional de Saúde da Família engajou-se com as equipes de saúde da UBS Padovani/Vista Bela - com destaque para a atuação dos ACS - na divulgação de informações sobre as formas de proteção contra a contaminação, orientações a usuários com risco de Covid-19, novos modos de funcionamento da UBS e o combate à circulação de notícias mentirosas nas redes sociais (fake news).

Elaboraram informativos e distribuíram em grupos de WhatsApp/Facebook de usuários, como grupo de compra e venda da região, grupo informativo do bairro, grupo da Atividade Física da UBS, grupos da igreja católica e da evangélica etc; colocando-se à disposição dos usuários para dúvidas. Um grupo de WhatsApp também foi criado com as principais lideranças comunitárias do território. Na medida em que informações, estudos e conclusões sobre a Covid-19 eram atualizadas, novos informativos foram produzidos e divulgados. Em parceria com usuários interessados, criaram o projeto "Se cuida, Vista Bela", concretizado a partir de verba doada por um projeto social de uma Igreja local. Foram produzidos e distribuídos 100 cartazes com informações pertinentes à pandemia em comércios, pontos de ônibus, prédios e postes, como também foram criados canais de comunicação via Whatsapp com a UBS. 

reorganização do trabalho do ACS. Neste estado, a principal forma de acesso à assistência à saúde é através da APS; caso da cidade de Coari, distante 363 quilômetros da capital Manaus e cujo acesso é por via fluvial e aérea. Em virtude das barreiras geográficas, a telessaúde encontrou obstáculos como a "indisponibilidade e/ou baixa qualidade de aparelhos, linhas telefônicas ou acesso à internet nas unidades e dos usuários, a questão da privacidade nos domicílios ou mesmo a capacidade de manuseio pelos pacientes" (Mata et al., 2020, p. 6). Apesar de tais dificuldades, todas as unidades do município foram equipadas com, no mínimo, um telefone móvel, com acesso à internet, recurso utilizado sempre que possível.

É neste contexto que o trabalho realizado pelos ACS nos territórios tem sua importância elevada. Mediante impossibilidades de contactar usuários de forma remota, os ACS realizavam VD (somente em casos de extrema necessidade) e busca ativa dos usuários em situação de risco, respeitando os protocolos sanitários. Assim, é importante situar que este protagonismo do trabalho dos ACS desenrola-se em um estado com um dos piores indicadores de morbimortalidade pelo novo coronavírus no Brasil (Mata et al, 2020), tendo vivido, em janeiro de 2021, o episódio de colapso do sistema de saúde, com a falta de oxigênio nos hospitais da capital Manaus gerando mortes e colocando em risco muitos doentes $(\mathrm{G} 1,2021)$.

28 Embora se identifique mais explícita e intensamente o uso de TICs durante a pandemia, destaca-se que essas ferramentas já eram regulamentadas pelo Ministério da Saúde anteriormente (Maciel et al., 2020). Mesmo assim, em muitos casos sua viabilidade no trabalho em saúde só foi possível com o uso dos aparelhos pessoais dos próprios trabalhadores, conforme atestam Bousquat et al. (2020, pp. 17-18) em sua pesquisa: "Chama a atenção a pequena disponibilidade de celulares institucionais, sendo que mais de $70 \%$ dos profissionais que responderam ao survey afirmaram usar o seu celular pessoal para contato com os usuários".

29 A implementação das TICs, principalmente no trabalho do ACS, categoria com menor visibilidade social na assistência, precisa ser tratada com cautela. É necessário acompanhar as mudanças efetivas provocadas nas práticas reais de trabalho, as exigências geradas, as construções sociotécnicas que as materializam, seus efeitos sobre a saúde e as dimensões sociais do trabalho (Baudin \& Nusshold, 2018).

30 E, de modo geral, as experiências de reorganização do trabalho na APS merecem melhor compreensão, tanto no que diz respeito ao seu alcance para cerceamento da pandemia, quanto aos efeitos à saúde dos trabalhadores em diferentes frentes de intervenção. 


\section{0 trabalho em saúde na APS e as reservas de alternativas}

31 Diante de nossa enorme tarefa planetária, muitos têm sinalizado para a importância de extrair aprendizados desta crise sanitária. No que diz respeito ao trabalho em saúde na APS, suas potencialidades não estão contidas apenas por suas atribuições prescritas, mas acima de tudo na forma como são concretizadas por seus trabalhadores e na capacidade de construção de um patrimônio coletivo. Assim, a indicação de aprendizados no debate público - como a valorização do SUS, o reconhecimento da importância do trabalho em equipe e da necessidade de avanço na qualificação dos trabalhadores - são pistas de reservas de alternativas (Schwartz, 2011) à espera de visibilidade e afirmação.

A atenção às reservas de alternativas deve ser acentuada. É alarmante que a existência do SUS e a experiência de APS já consolidada no Brasil não tenham conseguido produzir as melhores respostas em termos de prevenção e controle da Covid-19 (Giovanella et al., 2021). Entre os problemas na resposta dos sistemas de saúde e da APS, Giovanella et al. (2021) associam "ao predomínio de enfoques biomédicos, individualistas e assistencialistas” (p. 21, tradução livre). A despeito desta análise, questiona-se: a subestimação e subutilização da APS também estariam relacionadas a dificuldades de construção e mobilização de patrimônios no SUS e na APS?

Sob este ângulo, considera-se relevante acrescentar outro aspecto: a própria invisibilidade da atividade implicada na intencionalidade de transformar as ações de saúde na APS numa relação de serviço. $O$ encontro com os usuários é atravessado por dimensões parcialmente imprevisíveis e relacionadas à sua produção de normas de vida que evoca, de forma alargada, a dimensão gestionária do trabalho. Assim, o acesso às reservas de alternativas produzidas no SUS e na APS exige abordar o trabalho em saúde a partir do ponto de vista da atividade (Duraffourg, 2007/2010).

Neste horizonte, sugere-se o reposicionamento da capacitação de trabalhadores, educação permanente e gerenciamento de unidades de saúde através de sua instrução a partir do ponto de vista da atividade, isto é, na direção da ergoformação e do ergogerenciamento (Schwartz, 2011). Tais ações favoreceriam a emergência das escolhas e dos valores presentes no processo de reelaboração das normas antecedentes, a fim de superar suas lacunas. Se "a saúde começa com a tentativa de redesenhar parcialmente o meio em que se vive, em função de suas próprias normas" (Schwartz, 2011, p.139), este reposicionamento contribui para que os trabalhadores tenham, também eles, sua saúde afirmada e fortalecida (Brito, 2017).

\section{BIBLIOGRAFIA}

Baudin, C., \& Nusshold, P. (2018). Editorial. Laboreal, 16(2). https://doi.org/10.4000/laboreal.16773 
Bousquat, A., Nedel, F., Lima, J., Giovanella, L., Medina, M., Mendonça, M., ... \& Aquino, R. (2020). Desafios da atenção básica no enfrentamento da pandemia da Covid-19 no SUS (Relatório de Pesquisa/ 2020). Rio de Janeiro, Rede de Pesquisa em APS Abrasco. Recuperado de https://redeaps.org.br/ wp-content/uploads/2020/08/RelatorioDesafiosABCovid19SUS.pdf

Brito, J. (2017). Saúde: uma relação com o meio e os modos de vida. Laboreal, 13(1). https:// doi.org/10.4000/laboreal.2018

Cardona Jr., A., Andrade, C., \& Caldas, L. (2020). Educação em saúde: programa e canal de comunicação via WhatsApp da unidade básica de saúde do N6 para comunidade rural do sertão pernambucano. APS em Revista, 2(2). https://doi.org/10.14295/aps.v2i2.92

Conselho Federal de Enfermagem (2021a, 8 de janeiro). Brasil representa um terço das mortes de profissionais de enfermagem por Covid-19. Conselho Federal de Enfermagem. Disponível em http:// www.cofen.gov.br/brasil-responde-por-um-terco-das-mortes-de-profissionais-de-enfermagempor-covid-19_84357.html

Conselho Federal de Enfermagem (2021b, 9 de março). Brasil perde ao menos um profissional de saúde a cada 19 horas para a Covid. Conselho Federal de Enfermagem. Disponível em http:// www.cofen.gov.br/brasil-perde-ao-menos-um-profissional-de-saude-a-cada-19-horas-para-acovid_85778.html

Dantas, A. (2020). Coronavírus, o pedagogo da catástrofe: lições sobre o SUS e a relação entre público e privado. Trabalho, Educação e Saúde, 18(3). https://doi.org/10.1590/1981-7746-sol00281

Daumas, R., Silva, G., Tasca, R., Leite, I., Brasil, P., Greco, D., Grabois, V., \& Campos, G. (2020). O papel da atenção primária na rede de atenção à saúde no Brasil: limites e possibilidades no enfrentamento da COVID-19. Cadernos de Saúde Pública, 36(6). https://doi.org/ $10.1590 / 0102-311 \times 00104120$

Duraffourg, J. (2007/2010). O trabalho e o ponto de vista da atividade. In Y. Schwartz, \& L. Durrive (Eds.), Trabalho e Ergologia: conversas sobre a atividade humana (pp. 25-46). Niterói: EdUFF.

Fassa, A. C. (2021). "Temos que aproveitar esse momento de recrudescimento para avaliar onde estamos errando". Entrevista por Julia Neves. POLI: saúde, educação e trabalho, 13(75), 18-21. Disponível em http://www.epsjv.fiocruz.br/sites/default/files/poli_75_web_4.pdf

Favoreto, C. (2020). Atenção primária forte: elemento central no combate à pandemia de CovID-19. In C. Teixeira, C. Favoreto, D. Santos, L. Savassi, M. Guilam, M. Machado, \& M. Pinto (Eds.), COVID-19 e atenção primária: as experiências nos territórios (pp. 14-19). Recuperado de https:// profsaude-abrasco.fiocruz.br/sites/default/files/publicacoes/livro_-_covid-19_e_aps.pdf

G1 (2021, 14 de janeiro). Covid-19: Manaus vive colapso com hospitais sem oxigênio, doentes levados a outros estados, cemitérios sem vagas e toque de recolher. G1, Amazonas. Disponível em https:// g1.globo.com/am/amazonas/noticia/2021/01/14/covid-19-manaus-vive-colapso-com-hospitaissem-oxigenio-doentes-levados-a-outros-estados-cemiterios-sem-vagas-e-toque-derecolher.ghtml

Gamba, E., \& Righetti, S. (2021, 9 de abril). Mais de 500 mil pessoas que receberam a $1^{\text {a }}$ dose da vacina contra a Covid no Brasil não tomaram a $2^{\mathrm{a}}$. Folha de São Paulo. Recuperado de https:// www1.folha.uol.com.br/equilibrioesaude/2021/04/mais-de-500-mil-pessoas-que-receberama-1a-dose-da-vacina-contra-a-covid-no-brasil-nao-tomaram-a-2a.shtml

Giovanella, L., Vega, R., Tejerina-Silva, H., Acosta-Ramirez, N., Parada-Lezcano, M., Ríos, G., ... \& Feo, O. (2021). ¿Es la atención primaria de salud integral parte de la respuesta a la pandemia de 
Covid-19 en Latinoamérica? Trabalho, Educação e Saúde, 19. https://doi.org/10.1590/1981-7746solo0310

Guimarães, C. (2020, 25 de março). A importância de um sistema de saúde público e universal no enfrentamento à epidemia. Fundação Oswaldo Cruz, Escola Politécnica de Saúde Joaquim Venâncio. Disponível em https://www.epsjv.fiocruz.br/noticias/reportagem/a-importancia-de-umsistema-de-saude-publico-e-universal-no-enfrentamento-a

Jackson, J. M., Assunção, A., Algranti, E., Garcia, E., Saito, C., \& Maeno, M. (2020). A saúde do trabalhador e o enfrentamento da COVID-19. Revista Brasileira de Saúde Ocupacional, 45(e14). https://doi.org/10.1590/2317-6369ed0000120

Maciel, F., Santos, H., Carneiro, R, Souza, E., Prado, N., \& Teixeira, C. (2020). Agente comunitário de saúde: reflexões sobre o processo de trabalho em saúde em tempos de pandemia de Covid-19. Ciência \& Saúde Coletiva, 25(2). https://doi.org/10.1590/1413-812320202510.2.28102020

Mata, M., Castro, D., Gomes, C., Macedo, J., Checchi, M., Gama, A., \& Souza, L. (2020). A experiência da reorganização da atenção primária à saúde - APS e trabalho dos agentes comunitários de saúde frente à COVID-19 em um município no interior do Amazonas. Journal of Management \& Primary Health Care, 12(40). https://doi.org/10.14295/jmphc.v12.1014

Medina, M., Giovanella, L., Bousquat, A., Mendonça, M., \& Aquino, R. (2020). Atenção primária à saúde em tempos de COVID-19: o que fazer? Cadernos de Saúde Pública, 36(8). https://doi.org/ 10.1590/0102-311x00149720

Nogueira, M., Borges, C., Lacerda, A., Fonseca, A., Vellasques, A. , ... \& Rego, S. (2020). $1^{\circ}$ Boletim da pesquisa Monitoramento da saúde dos ACS em tempos de Covid-19. Rio de Janeiro: Fiocruz.

Portaria nํ․ 2.436, de 21 de setembro de 2017 (2017). Aprova a Política Nacional de Atenção Básica, estabelecendo a revisão de diretrizes para a organização da Atenção Básica, no âmbito do Sistema Único de Saúde. Recuperado de https://bvsms.saude.gov.br/bvs/saudelegis/gm/2017/ prt2436_22_09_2017.html

Riva, C., Moreira, K., Silva, E., \& Oliveira, J. (2020). Organizando fluxos no contexto da COVID-19: vivências em uma equipe no norte do país. In C. P. Teixeira, C. Favoreto, D. Santos, L. Savassi, M. Guilam, M. Machado, \& M. Pinto (Eds.), COVID-19 e atenção primária: as experiências nos territórios (pp. 21-25). Mestrado Profissional em Saúde da Família - PROFSAÚDE.

Salini, D., Jaramillo, J., Goudeaux, A., \& Poizat, G. (2018). Profesiones de servicio y digitalización: implicaciones y sugerencias para la concepción de procesos de formación. Laboreal, 14(2). https:// doi.org/10.4000/laboreal.584

Schmitt, G. (2021, 26 de janeiro). Quase mil profissionais de saúde morreram por Covid-19 no Brasil. o Globo. Disponível em https://oglobo.globo.com/sociedade/quase-mil-profissionais-desaude-morreram-por-covid-19-no-brasil-24853318

Schwartz, Y. (2011). Manifesto por um ergoengajamento. In P. F. Bendassolli, \& L. A. Soboll (Orgs.), Clínicas do trabalho: novas perspectivas para compreensão do trabalho na atualidade. São Paulo: Atlas.

Schwartz, Y., Adriano, R., \& Abderrahmane, F. (2008). Revisitar a actividade humana para colocar as questões do desenvolvimento: projecto de uma sinergia franco-lusófona. Laboreal, 4(1). https:// doi.org/10.4000/laboreal.12192

Seixas, C., Merhy, E., Feuerwerker, L., Santo, T., Slomp, H., \& Cruz K. (2021). A crise como potência: os cuidados de proximidade e a epidemia pela Covid-19. Interface (Botucatu), 25(1). https://doi.org/10.1590/interface.200379 
Teixeira, C., Soares, C., Souza, E., Lisboa, E., Pinto, I., Andrade, L., \& Espiridião, M. (2020). A saúde dos profissionais de saúde no enfrentamento da pandemia de Covid-19. Ciência \& Saúde Coletiva, 25(9), 3465-3474. https://doi.org/10.1590/1413-81232020259.19562020

Teixeira, M., Medina, M.., Costa, M., Barral-Netto, M., Carreiro, R., \& Aquino, R. (2020).

Reorganização da atenção primária à saúde para vigilância universal e contenção da COVID-19. Epidemiologia e Serviços de Saúde, 29(4), 1-5. https://doi.org/10.5123/s1679-49742020000400015

Zarifian, P. (2001). Objetivo competência: por uma nova lógica. São Paulo: Atlas.

\section{AUTOR}

\section{ANA CLÁUDIA BARBOSA DA SILVA-ROOSLI}

https://orcid.org/0000-0003-3795-9357

Departamento de Psicologia Social e Institucional da Universidade Estadual de Londrina (UEL), Rodovia Celso Garcia Cid, PR 445 Km 380, Campus Universitário, 86057-970 Londrina-PR, Brasil anaclaudia79@uel.br 